\title{
Actualización de contenidos en el campo disciplinar de matemáticas del componente propedéutico del bachillerato tecnológico: el caso de las funciones especiales
}

\begin{abstract}
Updating of contents in the disciplinary field of mathematics of technological high school: the case of special functions
\end{abstract}

Atualização de conteúdos no campo disciplinar de matemática da componente preparatória do bacharelado tecnológico: o caso das funções especiais

Mario. A. Sandoval-Hernández* Instituto Tecnológico Superior de Poza Rica, Tecnológico Nacional de México, México mario.sandoval@itspozarica.edu.mx

Centro de Bachillerato Tecnológico industrial y de servicios 190, México marioalberto.sandoval.cb190@dgeti.sems.gob.mx https://orcid.org/0000-0002-5518-3858

Sergio Hernández-Méndez Universidad de Xalapa, Escuela de Ingeniería, México sergio.h@ux.edu.mx https://orcid.org/0000-0001-9997-9690

Salvador E. Torreblanca-Bouchan

Centro de Bachillerato Tecnológico industrial y de servicios 190, México salvadorenrique.torreblanca.cb190@dgeti.sems.gob.mx https://orcid.org/0000-0002-1695-2933 

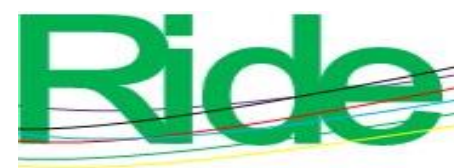

Revista Iberoamericana para la Investigación y el Desarrollo Educativo ISSN $2007-7467$

Gerardo U. Díaz-Arango Instituto Tecnológico Superior de Poza Rica, Tecnológico Nacional de México, México gerardo.diaz@itspozarica.edu.mx https://orcid.org/0000-0002-8144-6615

*Autor corresponsal.

\section{Resumen}

El estudio de las funciones en el bachillerato en México se ha limitado únicamente a las elementales ignorando a las funciones hiperbólicas y especiales. Estas últimas, por lo general, se encuentran en términos de integrales, y para evaluarlas deben utilizarse algoritmos numéricos que se hallan fuera del alcance de los estudiantes de este nivel educativo. Por tanto, en este artículo se propone actualizar los contenidos en la asignatura propedéutica Matemáticas Aplicadas del campo disciplinar de matemáticas del bachillerato tecnológico en la que se agrega la función Lambert W al estudio de las funciones trascendentes. Además, se considera adicionar el estudio de las funciones hiperbólicas y especiales a través de aproximaciones matemáticas que se encuentran en términos de funciones elementales, lo que facilitará su implementación en software educativo como GeoGebra. Asimismo, se ofrecen cuatro ejemplos en donde se aplican las aproximaciones de las funciones que se consideran en la propuesta curricular, las cuales pueden servir de guía para mostrar aplicaciones relacionadas con la tecnología y el cotidiano de los alumnos.

Palabras clave: discurso matemático escolar, el cotidiano, graficado y aplicación de funciones matemáticas.

\footnotetext{
Abstract

The study of functions in high school has been limited only to elementary ones, ignoring hyperbolic and special functions. The latter are generally found in terms of integrals and numerical algorithms must be used to evaluate them, leaving them out of the reach of high school students. In this paper a content update is made in the course of applied mathematics of the high school in which the Lambert $\mathrm{W}$ function is added to the study of transcendent functions. In the same way, the curricular proposal considers the study of hyperbolic functions and special functions through mathematical approximations found in terms of
} 

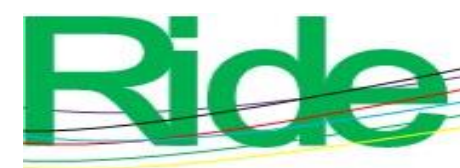

Revista Iberoamericana para la Investigación y el Desarrollo Educativo

ISSN $2007-7467$

elementary functions, which facilitates their implementation in educational software such as GeoGebra. In addition, in this work four examples are presented where the approximations of the functions that are considered in the curricular proposal the functions are applied and that can serve as a guide to show applications related to technology and the daily life of the students.

Keywords: school math speech, daily life, graphing matematical functions.

\section{Resumo}

O estudo das funções no ensino médio no México foi limitado apenas aos elementares, de modo que as funções hiperbólicas e especiais foram ignoradas. Estes últimos são geralmente encontrados em termos de integrais, e algoritmos numéricos que estão além do alcance dos alunos neste nível educacional devem ser usados para avaliá-los. Portanto, neste artigo propõe-se a atualização dos conteúdos da disciplina preparatória de Matemática Aplicada do campo disciplinar da matemática do bacharelado em tecnologia em que a função de Lambert W é agregada ao estudo das funções transcendentes. Além disso, considera-se agregar o estudo de funções hiperbólicas e especiais por meio de aproximações matemáticas encontradas em termos de funções elementares, o que facilitará sua implementação em softwares educacionais como o GeoGebra. Da mesma forma, são oferecidos quatro exemplos onde se aplicam as aproximações das funções consideradas na proposta curricular, que podem servir como um guia para mostrar aplicações relacionadas à tecnologia e ao cotidiano dos alunos.

Palavras-chave: discurso matemático escolar, cotidiano, representação gráfica e aplicação de funções matemáticas.

Fecha Recepción: Marzo 2021

Fecha Aceptación: Septiembre 2021 

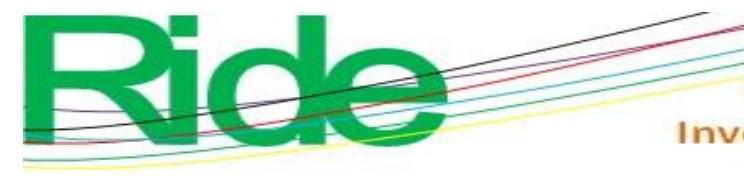

Investigación y el Desarrollo Educativo ISSN 2007 - 7467

\section{Introducción}

El uso de las funciones cobra importancia en la ciencia y en la ingeniería, ya que la representación de cualquier modelo, fenómeno o sistema se realiza mediante ecuaciones estáticas (Baldor, 2011), Barnett (1994), ecuaciones diferenciales (Edwards y Penney, 2011), Zill (1997) e integro-diferenciales (Wazwaz, 2011), mientras que el comportamiento de estos fenómenos o sistemas se pueden expresar a través de funciones algebraicas y trascendentes, que dan lugar a una representación gráfica (Barnett, 1994). Sin la existencia de las funciones sería imposible realizar algún tipo de análisis, como lo son los análisis de estabilidad, respuesta en frecuencia (Ogata ,2003), entre otras.

Desde hace años —y antes de la reforma de 2004 en el sistema del bachillerato tecnológico de la Dirección Tecnológica industrial (DGETI) en México (DOF, 2004) el estudio de las funciones trigonométricas (Barnett, 1994) se comenzaba a estudiar en segundo semestre (DOF, 2004, 2012) en la asignatura de geometría y trigonometría; en tercer semestre en geometría analítica (DOF, 2004, 2012; Kindle, 1994), y en cuarto semestre en la asignatura de cálculo diferencial (Ayres, Mendelson y Abellanas, 1994), (Casares, 2018); DOF, 2004, 2012). Asimismo, cálculo integral se cursa en quinto semestre, donde se presentan los diferentes métodos de integración, la integral definida y sus aplicaciones, mientras que en sexto semestre se estudia la asignatura de probabilidad y estadística (DOF, 2012). En ese mismo semestre, se puede cursar la asignatura de matemática aplicada, que también forma parte del campo disciplinar de matemáticas, propedéutica y a su vez optativa (DOF, 2012). Esta cátedra está dirigida a los alumnos que desean continuar sus estudios en el nivel universitario. En matemáticas aplicadas se estudian la modelación matemática, el cálculo y las relaciones trascendentes. El actual programa de estudios de la asignatura Matemáticas Aplicadas tiene la deficiencia de abordar de manera insuficiente la modelación utilizando funciones, ya que se limita a los ejemplos clásicos, con una visión muy fragmentada de la realidad (DOF, 2012). 


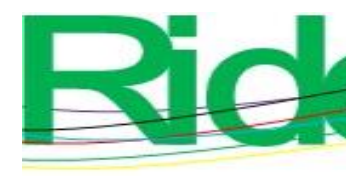

Revista Iberoamericana para la
Investigación y el Desarrollo Educativo
ISSN $2007-7467$

\section{Discurso matemático escolar}

El discurso matemático escolar (DME) es todo el lenguaje que se introduce en una clase, donde los estudiantes hablan, exponen sus conceptos matemáticos, discuten ideas y solución de problemas (Soto, Gómez, Silva y Cordero, 2012). Se caracteriza por ser hegemónico, utilitario y desprovisto de marcos de referencia, con lo cual impone significados, argumentos y procedimientos centrados en los objetos matemáticos (Soto et al., 2012; Soto y Cantoral 2014).

Una manera de visualizar al DME es identificando todo aquello que permanece a pesar de las innovaciones en relación con la matemática escolar debido a que dicha innovación, en el fondo, no modifica aquello que se está enseñando, sino solo cómo se está enseñando. Es importante advertir que muchos libros de texto de matemáticas utilizados en el aula se encuentran concebidos bajo el DME (Uriza, Espinosa y Gasperini, 2015), el cual influye en los procesos de enseñanza-aprendizaje.

\section{El cotidiano}

De manera tradicional, la enseñanza de las matemáticas se ha hecho de forma descontextualizada, carente de un significado y ajena al mundo real, pues se ha soslayado que su objetivo es resolver problemas del cotidiano y, en su caso, de la ciencia, de ahí que se deban intentar resolver preguntas como las siguientes: ¿esto para qué me sirve?, ¿dónde lo voy a utilizar?

En tal sentido, el cotidiano del alumno, por lo general, lo vinculamos en la escuela a situaciones personales, donde hay algún tipo de intercambio. Por eso, en los libros de texto (Olguín-Díaz y Sánchez-Linares, 2016) es frecuente hallar ejercicios típicos como los de Rosita, Pepito y Juanita, quienes fueron a la tienda de la esquina a comprar 5 paletas, un paquete de galletas y uno de chicles, que pagaron con un billete de $\$ 50$ y están interesados en saber cuánto les darán de cambio.

No obstante, esta concepción es limitada porque el uso de funciones no debe restringirse exclusivamente a modelos y situaciones que se le presentan al alumno dentro del salón de clases. Algunos ejemplos de aplicaciones típicas que se le suelen ofrecer al estudiante son el crecimiento de una población en función del tiempo o el representar gráficamente la relación del costo de un producto contra su demanda, entre otros. Sin 


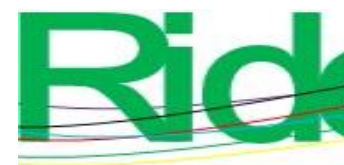

Revista Iberoamericana para la Investigación y el Desarrollo Educativo ISSN 2007 - 7467

embargo, al presentar únicamente estos problemas tradicionales en el salón, se fomenta de nuevo el DME porque pareciera que las matemáticas solo se ajustan al ámbito interpersonal.

En cambio, el cotidiano que rodea al estudiante es muy amplio, pues no solo incluye intercambios monetarios, sino también fenómenos físicos, químicos y tecnológicos. Por eso, a los alumnos les podría interesar otros fenómenos, como el pronóstico del estado del tiempo de la zona donde vive, la propagación de alguna enfermedad, la innovación en el área de las ciencias de la salud (Cordero, 2013; Vazquez-Leal et al., 2015b), los efectos de la temperatura sobre los materiales de construcción (Treviño et al., 2004), la composición de los productos de limpieza (Collado, Huallapacusi y Osores, 2005), las ondas electromagnéticas emitidas y recibidas por aparatos electrónicos (p. ej., hornos, teléfonos celulares, radio, televisión) (Proakis y Masoud, 2001; Haykin, 1994), el comportamiento de las vibraciones en un puente con estructura metálica (Bermúdez, 2005), entre otros. En consecuencia, resulta pertinente que el estudiante disponga de un panorama más amplio de la aplicación de las funciones para resolver no solo situaciones cotidianas, sino también para comprender la tecnología que existe a su alrededor.

\section{Metodología}

En este trabajo se realizó una investigación documental en los programas de estudio del campo disciplinar de matemáticas en estructuras curriculares del bachillerato tecnológico con el fin de conocer las actualizaciones en los contenidos programáticos entre los acuerdos 345 y 653 en las bases de datos del Diario Oficial de la Federación y en la página web de la Coordinación Sectorial de Fortalecimiento Académico en México (COSFAC).

La investigación incluyó más de 60 libros de precálculo, cálculo y áreas afines de la ingeniería (de diferentes autores y editoriales en los idiomas inglés y español) para comparar los contenidos publicados. En algunos libros (como los de precálculo publicados por un mismo autor) se han contrastado las ediciones actualizadas y previas para verificar los cambios efectuados. Entre los libros revisados se encontraron manuales de funciones matemáticas, tales como los de Abramowitz, Stegun y Romer (1988) y Oldham, Myland y Spanier (2010), en los cuales se buscaron las funciones especiales. En las referencias de este artículo, se ha considerado citar los libros de autores más conocidos en los que se ha hecho la revisión de los contenidos. 


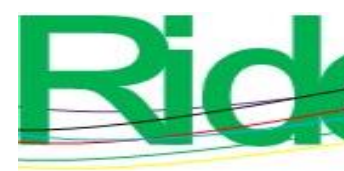

Revista Iberoamericana para la
Investigación y el Desarrollo Educativo
ISSN $2007-7467$

Asimismo, se consultaron bases de datos tales como Google Scholar, Scielo, Dialnet, Journal Citation Reports y Scimago. Los criterios de búsqueda fueron artículos científicos de aproximaciones y aplicaciones de las funciones especiales, con palabras clave como special functions, approximative expresions for special functions y special functions applied.

A partir de lo anterior, se elaboró una base de datos en Excel en donde se registraron aproximadamente 40 artículos relacionados con las funciones especiales, sus aplicaciones, las aproximación de funciones y métodos asintóticos (ya que en estos se basan las aproximaciones matemáticas para estas funciones y en general cuando se propone una solución aproximativa para una ecuación diferencial), los cuales se clasificaron en a) funciones especiales y b) aplicaciones en la ciencia y tecnología. De esta clasificación se consideró la viabilidad para implementar en el bachillerato y la facilidad para desarrollar las funciones en cualquier software educativo.

\section{Materiales}

Para la presentación de las gráficas y ejemplos resueltos presentados en este trabajo se empleó el software matemático Maple 2015. Asimismo, se utilizó una computadora con sistema operativo Linux Ubuntu (versión 18.04.5 LTS) con procesador Intel I7-7700@ $3600 \mathrm{GHz} \times 8$.

\section{Resultados}

En la investigación documental se encontró que en las nuevas ediciones de algunos libros de texto (Baldor, 2011; Barnett, 1994; Edwards y Penney, 2011; Kindle, 1994; Leithold, 2012; Ogata, 2003; Olvera, 1991; Purcell, Rigdon y Varbeg, 2007; Stewart, 2015; Wazwaz, 2011; Zill, 1997) se realizan cambios favorables en la pedagogía con que se presentan los conceptos temáticos, con más ejemplos demostrativos y con un mayor número de ejercicios propuestos. En otros casos, los textos incorporan el uso de software matemático como Maple (Fox, 2011), Matlab (Almenar, Isla, Gutiérrez y Luege, 2018), GNU Octave (Lie, 2019), GeoGebra (Mora-Sánchez, 2019), Excel (Torres-Remon, 2016), entre otros.

Una situación análoga ocurre en los programas de estudio de la COSFAC cuando se hace una "revisión" de contenidos en asignaturas de tronco común y especialidad (DOF, 2004, 2012), ya que por lo general se enfocan más en el diseño de estrategias de aprendizaje, 


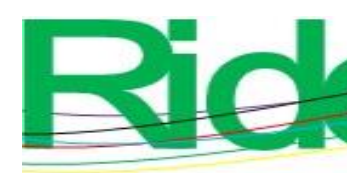

Revista Iberoamericana para la
Investigación y el Desarrollo Educativo
ISSN $2007-7467$

de evaluación y de planeación didáctica (Arceo, Rojas y González, 2010; Charur, 2016; Morales-Lizama, 2016), y del uso de las tecnologías de la información y de la comunicación (Álvarez y Mayo, 2009). Sin embargo, en programas de estudio del campo disciplinar de matemáticas, en estructuras curriculares del bachillerato tecnológico de los acuerdos 345 y 653, no se propuso la introducción de contenidos nuevos dentro del campo disciplinar de matemáticas. Además, se encontró que el estudio de las funciones en el bachillerato se ha limitado únicamente a las algebraicas y trascendentes.

En referencia a las funciones especiales, se halló que son muy importantes en los análisis matemáticos de la ciencia y la ingeniería, ya que cada una de las distintas funciones tiene diversas aplicaciones en diferentes ramas de la ciencia. Por ejemplo, las funciones de Fresnel (Oldham et al., 2010) tienen aplicaciones en la óptica. Las funciones elípticas de primera y segunda clase se aplican en la mecánica celestial (Fukushima y Kopeikin, 2014), el electromagnetismo (Brizard, 2009; Greenhill, 1907), y las órbitas planetarias (Fukushima y Kopeikin, 2014). La función error la encontramos aplicada en fenómenos de transporte (Bird, Stewart y Lightfoot, 2002) y comunicaciones digitales (Sadiku, 1998), mientras que la función de densidad de probabilidad en la estadística la hallamos en las áreas de ingeniería (González y Woods, 1996), ciencias sociales (Hernández-Sampieri, Fernández-Collado y Baptista, 2010), ciencias de la salud (Wayne, 2000), entre otros.

En la tabla 1 se resumen algunas de las funciones especiales utilizadas en la ciencia y la ingeniería. Se presentan sus nombres, símbolos, algunas de sus aplicaciones, su clasificación y las referencias donde se encuentran. Estas funciones tienen un alto potencial de ser incluidas en la asignatura de matemática aplicada del bachillerato tecnológico, ya que poseen muchas aplicaciones relacionados con el cotidiano del alumno.

De la misma forma, en dicha tabla se han agregado las funciones hiperbólicas. En la investigación llevada a cabo encontramos que los libros de texto del nivel medio superior no las toman en cuenta, ya que centran su atención en la derivación de funciones algebraicas y trascendentes. Sin embargo, las reglas de derivación para estas funciones son prácticamente las mismas. 
Tabla 1. Funciones especiales y sus aplicaciones

\begin{tabular}{|c|c|c|c|c|}
\hline $\begin{array}{l}\text { Nombre de la } \\
\text { función }\end{array}$ & Símbolo & Aplicaciones & Clasificación & Referencia \\
\hline Lambert W & $\mathrm{W}(x)$ & Electrónica, química & Trascendente & $\begin{array}{l}\text { Corless et al. (1996), } \\
\text { Johansson (2020) }\end{array}$ \\
\hline $\begin{array}{l}\text { Función } \\
\text { signo }\end{array}$ & $\operatorname{sgn}(x)$ & Análisis de funciones & Especial & $\begin{array}{l}\text { Abramowitz et al. (1988), } \\
\text { Sandoval-Hernández et al. } \\
\text { (2018) }\end{array}$ \\
\hline $\begin{array}{l}\text { Integrales de } \\
\text { Fresnel }\end{array}$ & $\mathrm{S}(x), \mathrm{C}(x)$ & $\begin{array}{l}\text { Óptica, } \\
\text { electromagnetismo }\end{array}$ & Especial & $\begin{array}{lcc}\text { Hecht y } & \text { Zajac } & \text { (1987), } \\
\text { Balassone } & y & \text { Romero } \\
(2015) & & \end{array}$ \\
\hline Función error & $\operatorname{erf}(x)$ & $\begin{array}{l}\text { Comunicaciones, } \\
\text { fenómenos de } \\
\text { transporte }\end{array}$ & Especial & $\begin{array}{l}\text { Bird et al., (2002), Proakis } \\
\text { y Masoud (2001), } \\
\text { Haykin (1994) }\end{array}$ \\
\hline $\begin{array}{l}\text { Función de } \\
\text { distribución } \\
\text { acumulativa } \\
\text { de } \\
\text { probabilidad }\end{array}$ & $\mathrm{p}(x)$ & Estadística & Especial & $\begin{array}{l}\text { González y Woods (1996), } \\
\text { Hernández-Sampieri et al. } \\
\text { (2010) } \\
\text { Wayne ( 2000) }\end{array}$ \\
\hline $\begin{array}{l}\text { Funciones } \\
\text { elípticas }\end{array}$ & $\mathrm{F}(x), \mathrm{E}(x)$ & $\begin{array}{l}\text { Mecánica, teoría } \\
\text { electromagnética }\end{array}$ & Especial & $\begin{array}{l}\text { Fukushima y Kopeikin } \\
(2014)\end{array}$ \\
\hline $\begin{array}{l}\text { Función } \\
\text { Gamma }\end{array}$ & $\Gamma(x)$ & $\begin{array}{l}\text { Matemáticas, } \\
\text { estadística }\end{array}$ & Especial & Abramowitz et al. (1988) \\
\hline $\begin{array}{l}\text { Funciones } \\
\text { hiperbólicas }\end{array}$ & $\begin{array}{l}\text { sinh, cosh } \\
\text { tanh, csch } \\
\text { sech, tanh }\end{array}$ & $\begin{array}{l}\text { Mecánica, } \\
\text { electricidad, } \\
\text { matemáticas }\end{array}$ & $\begin{array}{l}\text { Combinación } \\
\text { de funciones } \\
\text { exponenciales }\end{array}$ & $\begin{array}{l}\text { Barnett (1994), } \\
\text { Leithold (2012), } \\
\text { Stewart (2015) }\end{array}$ \\
\hline
\end{tabular}

Fuente: Elaboración propia

Referente a la función Lambert $\mathrm{W}$, encontramos que actualmente los libros de trigonometría y precálculo no incluyen su estudio y ni siquiera mencionan su existencia. Actualmente, esta función es muy empleada en la solución de problemas de la ingeniería, por lo que se halla publicada en diversos artículos científicos. En Corless et al. (1996) y VazquezLeal et al. (2020) se consigue una breve reseña histórica de dicha función. 


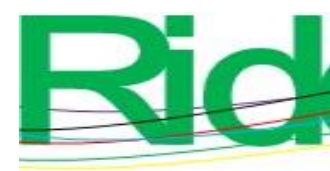

Revista Iberoamericana para la Investigación y el Desarrollo Educativo ISSN 2007 - 7467

En la tabla 2 se ofrece el catálogo de funciones propuestas para la asignatura de matemática aplicada, en donde se presenta el tipo de función por utilizar, ya sea exacta o aproximativa, así como la referencia de donde se han obtenido.

Tabla 2. Funciones especiales y sus aproximaciones

\begin{tabular}{|c|c|c|}
\hline Nombre de la función & Tipo de expresión & Publicado en \\
\hline Lambert W & Aproximación & Vázquez-Leal et al. (2019) \\
\hline Función signo & Función exacta & Abramowitz et al. (1988) \\
\hline Integrales de Fresnel & Aproximación & Sandoval-Hernández et al. (2018) \\
\hline Función error & Aproximación & Sandoval-Hernández et al. (2019a) \\
\hline $\begin{array}{l}\text { Función de densidad de } \\
\text { probabilidad acumulativa }\end{array}$ & Aproximación & Sandoval-Hernández et al. (2019a) \\
\hline $\begin{array}{l}\text { Funciones elípticas de } \\
\text { primera y segunda clase }\end{array}$ & $\begin{array}{l}\text { Aproximación, } \\
\text { Metodología }^{*}\end{array}$ & $\begin{array}{l}\text { Vázquez-Leal y Sarmiento-Reyes (2015b) } \\
\text { Sandoval-Hernández et al. (2019b) }\end{array}$ \\
\hline Función Gamma & $\begin{array}{l}\text { Aproximación, } \\
\text { Metodología* }\end{array}$ & $\begin{array}{l}\text { Zhen-Han y Jing-Feng (2018) } \\
\text { Komla-Amenyou (2018) } \\
\text { Vázquez-Leal y Sarmiento-Reyes (2015b) } \\
\text { Vázquez-Leal et al. (2019) } \\
\text { Sandoval-Hernández et al (2019a) }\end{array}$ \\
\hline Funciones hiperbólicas & Función exacta & Barnett (1994) \\
\hline \multicolumn{3}{|c|}{$\begin{array}{l}\text { *Nota: Aproximaciones obtenidas con las metodologías matemáticas aproximativas publicadas } \\
\text { según la referencia. }\end{array}$} \\
\hline
\end{tabular}

Fuente: Elaboración propia

Las aproximaciones presentadas en las referencias contenidas en la tabla 2 se han seleccionado porque están en términos de funciones elementales, lo que facilita implementarlas en cualquier software educativo haciendo posible su estudio en el aula. En la literatura existen más aproximaciones para las funciones de la tabla 2, aunque algunas de ellas se encuentran en términos de otras funciones especiales y desconocidas que dificultan su implementación en el bachillerato tecnológico haciendo uso de software matemático especializado. 


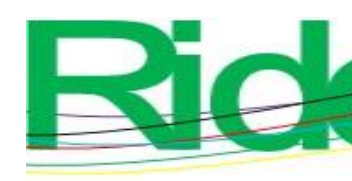

Revista Iberoamericana para la

Investigación y el Desarrollo Educativo

ISSN $2007-7467$

\section{La propuesta de actualización de contenidos}

En la figura 1 proponemos el nuevo contenido sugerido para la asignatura de matemática aplicada para que el alumno refuerce los conceptos relacionados al manejo de funciones y el modelado de situaciones del entorno que contribuyan a desarrollar las competencias disciplinares que forman parte del perfil del egresado de la EMS (DOF, 2008).

En el primer bloque se estudian las desigualdades y las funciones, en donde se recuperan los conocimientos de la recta numérica y el plano cartesiano. Además, se presenta el valor absoluto, los intervalos, el dominio y el rango de una función y las desigualdades. En el estudio de las funciones es necesario identificar el dominio y contradominio de una función, así como la manera de interpretarlos y representarlos algebraicamente y de forma gráfica. Además, se estudia el criterio de la línea vertical para identificar si una gráfica representa una función o una relación. También se estudia la periodicidad y paridad de una función.

En el segundo bloque se estudian las funciones trascendentes e hiperbólicas, así como las características de las funciones trigonométricas circulares, hiperbólicas, logarítmicas y exponenciales. Se ha agregado al grupo de funciones trascendentes la función Lambert W.

El tercer bloque presenta el grupo de las funciones especiales que incluye la función signo, funciones de Fresnel, función error y complementario, funciones elípticas, función de probabilidad normal y función Gamma. Además, se abre la posibilidad de incluir más funciones especiales.

En la propuesta que presentamos en este artículo se considera en todo momento la graficación de las funciones a lo largo del semestre. Se pretende que el alumno identifique e interprete las diferentes propiedades de las funciones como es la paridad, periodicidad de una función, así como las características de cada una de las funciones trascendentes. Por último, se hace énfasis en presentar y proponer a los alumnos diferentes casos de estudio, donde se incluya algún tipo de modelación utilizando alguna de las diferentes funciones trascendentes, hiperbólicas, especiales y algebraicas. 
Figura 1. Programa de estudios propuesto para la asignatura de matemáticas aplicadas

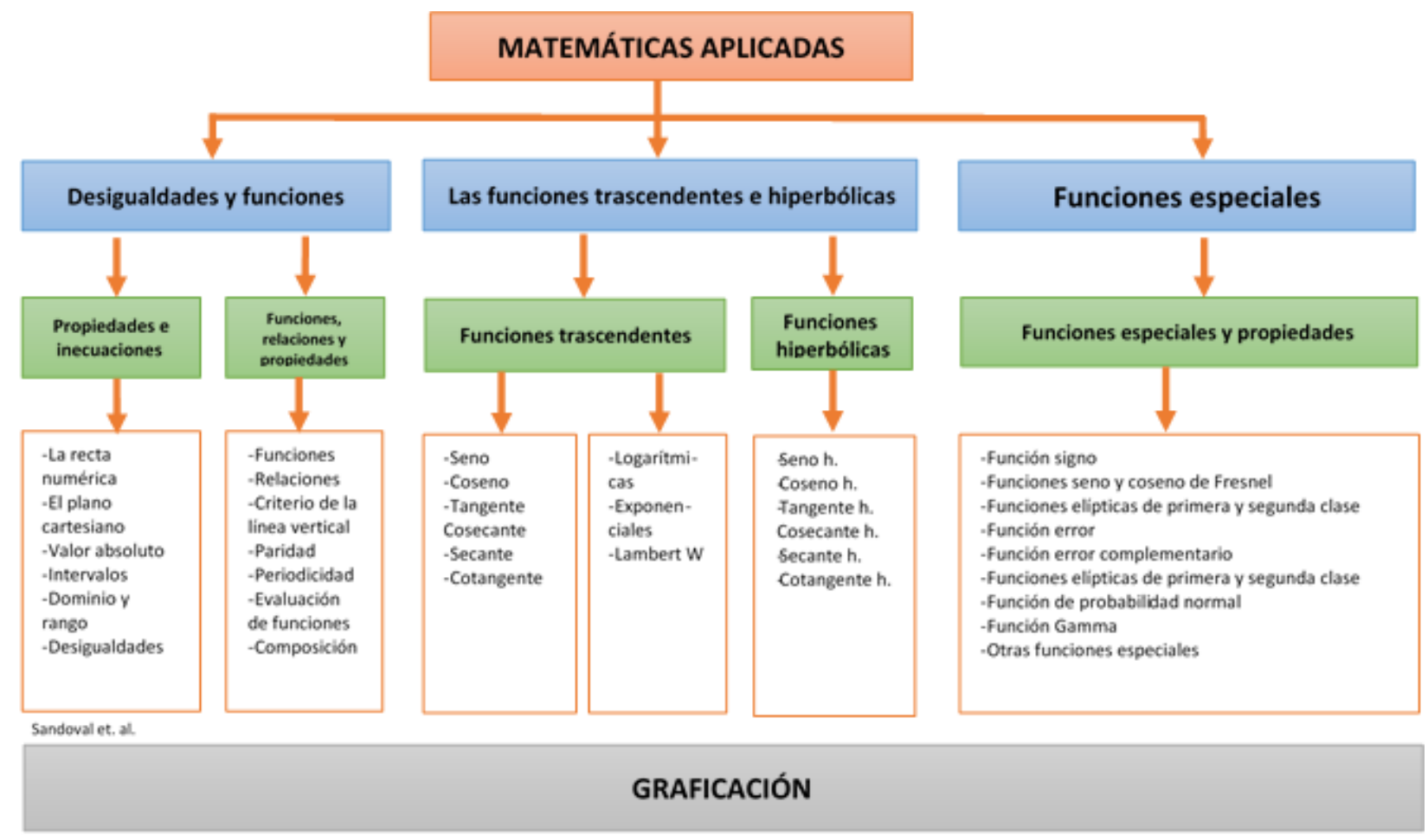

\section{APLICACIONES}

Modelación, solución de situaciones cotidianas inmediatas, en la tecnología, sistemas mecánicos, eléctricos, químicos, fenómenos físicos de la naturaleza, interpretación de gráficos

Fuente: Elaboración propia

\section{Discusión}

En general, en todos los niveles educativos (incluido el bachillerato tecnológico), es común el uso del libro de texto, el cual dispone de una amplia variedad y es divulgado por prestigiosas editoriales. Esta obra ha funcionado como guía y fuente de información adicional, y es un medio por el cual se construye el consenso educativo. "Sirve para introducir una ideología y para legitimar contenidos y formas específicas del conocimiento escolar” (Uriza et al., 2015). Sin embargo, en el área de la matemática educativa se han realizado diferentes propuestas para facilitar el entendimiento del manejo de las funciones. Por ejemplo, para comprender el comportamiento de una función, en Cordero Osorio (2002) se analiza que la situación linealidad del polinomio tiene la intencionalidad de relacionar la recta tangente con el comportamiento de la función. En el caso de la ecuación

$$
y=a x^{2}+b x+c
$$




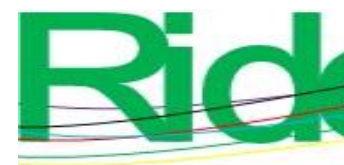

Revista Iberoamericana para la

Investigación y el Desarrollo Educativo

ISSN 2007 - 7467

los estudiantes deben construir un significado a la parte lineal del polinomio, es decir, para los términos lineal e independiente en relación con el comportamiento tendencial de la gráfica del polinomio (término cuadrático). La reconstrucción del significado consiste en dos aspectos: identificar la propiedad de linealidad y de establecerla como argumento (Uriza, 2000). De esta forma, la linealidad del polinomio consiste en que la parte lineal de cualquier polinomio

$$
P(x)=a_{n} x^{n}+a_{n-1} x^{n-1}+\cdots+a_{1} x+a_{0},(2)
$$

es la recta tangente al polinomio que pasa por el punto $(0, P(0))$ (Cordero Osorio, 2002).

Existen otras propuestas interesantes para el aprendizaje de las matemáticas. En Cordero Osorio y Domínguez García (2001) se publicó un diseño de situación de las asíntotas senoidales, tomando en cuenta las experiencias previas que tienen los estudiantes. En primer lugar, el conocimiento gráfico: "Determinar si las gráficas que a continuación se presentan tienen comportamiento asintótico". En segundo lugar, las experiencias algebraicas: "Relacionar las siguientes funciones con sus respectivas gráficas" y, tercero, la analítica: "Proporcionar una definición de la asíntota de una función. En Cordero y Suárez (2005) se ofrece un diseño didáctico que ejemplificó la modelación gráfica, en donde se resignificó la parábola y los modelos gráficos relacionados con situaciones del desplazamiento de una persona.

En los trabajos de Uriza (2000), Cordero Osorio (2009) y Cordero Osorio y Domínguez García (2001) se provee al estudiante de elementos que enriquecen la manera de comprender el comportamiento variacional de las funciones. Asimismo, el estudiante aprende a reconocer el comportamiento de la función, independientemente del tipo que se esté analizando, ya sea continua, discontinua, polinomial o trascendente. Sin embargo, en todos estos trabajos solo se aborda el estudio de las funciones elementales de manera algebraica y geométrica, ya que los análisis realizados se centran en estas dos características. Consideramos que estas propuestas didácticas que han propuesto diferentes autores deben ir acompañadas de situaciones físicas del cotidiano que sean significativas para los alumnos (Filobello-Nino et al., 2017a; Filobello-Nino et al., 2017b; Serway y Jewett, 2018; VázquezLeal et al., 2017). 


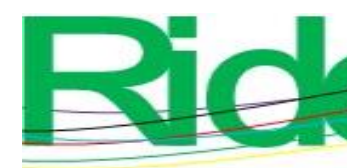

Revista Iberoamericana para la
Investigación y el Desarrollo Educativo

ISSN $2007-7467$

Es importante destacar que los libros de texto del bachillerato tecnológico tienden a hacer este tipo de análisis (algebraico y geométrico), aunque no alcanzan a superar completamente el DME porque durante mucho tiempo se ha soslayado el significado físico en el cotidiano. En consecuencia, la aplicación en nivel bachillerato de las matemáticas al cotidiano (Soto et al., 2012; Soto y Cantoral, 2014) puede ampliarse considerablemente.

\section{Las funciones especiales y el grupo de las funciones aproximadas}

En la educación media superior y en el bachillerato tecnológico, en años anteriores no ha sido posible incluir en los programas de estudio el comportamiento de las funciones especiales porque no es fácil evaluarlas o graficarlas simplemente con lápiz y papel o con software educativo sin contar con una expresión algebraica. Para este propósito incluimos en esta sección el conjunto de aproximaciones para las funciones especiales que se han propuesto en la asignatura de matemática aplicada.

La notación de estas aproximaciones tienen el símbolo de tilde ( ), aunque para efectos prácticos se consideran funciones. En esta sección se presenta su aproximación y su gráfica. Para mayores detalles se pueden consultar las referencias de este artículo.

\section{La función signo}

La función signo se define como

$$
\operatorname{sgn}(x)=\left\{\begin{array}{c}
1, \text { si }>0 \\
0, \text { si } x=0 \\
-1, \text { si } x<0
\end{array}\right.
$$

\section{La función Lambert W}

La función Lambert W se define como la inversa de la función trascendental

$$
y(x) \mathrm{e}^{y(x)}=x .
$$

Despejando se tiene

$$
y(x)=W(x)
$$

donde $x$ puede ser un número real o complejo y $W(x)$ es la función Lambert $\mathrm{W}$. Esta función pertenece al grupo de funciones trascendentes y es mutivaluada porque en el dominio de los números reales tiene dos ramas. En la figura se pueden apreciar las ramas $W_{0}(x)$ y $W_{-1}(x)$. 

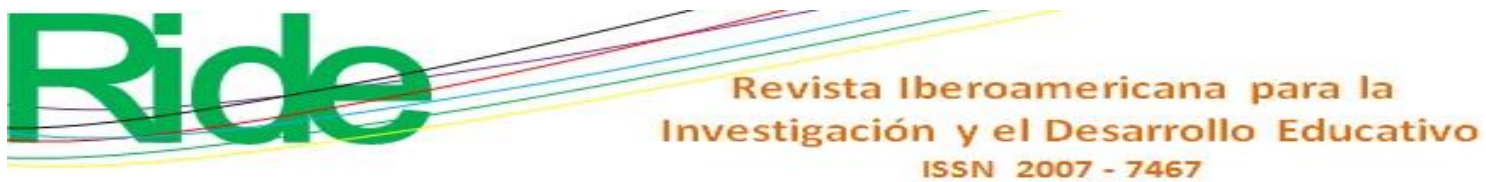

$W_{0}(x)$ es la rama principal que satisface la condición $W(x) \geq-1$. Esta rama se puede dividir en dos zonas $W_{0^{-}}(x)$ y $W_{0^{+}}(x)$, porque en este lugar la función es multivaluada. $W_{-1}(x)$ es la rama inferior y satisface la condición $W(x) \leq-1$. Las dos ramas de la función se encuentran en $(-1 / e,-1)$. De esta manera en el intervalo $-1 / e<x<0$ hay dos valores posibles de $W(x)$. El primero para $W_{0^{-}}(x)$ y el segundo para $W_{-1}(x)$. En la figura 2 se presentan las dos ramas de la función Lambert W.

Figura 2. Función Lambert W

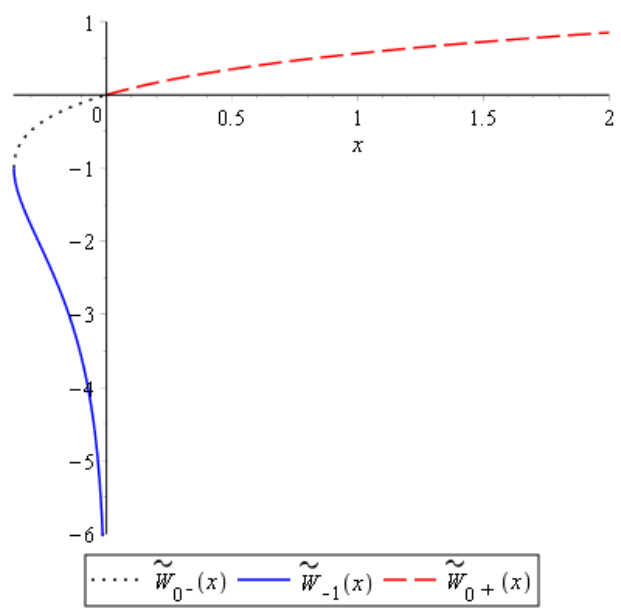

Fuente: Elaboración propia

En Vázquez-Leal et al. (2019) se propuso la aproximación para Lambert W para ambas ramas. El subíndice cero hace referencia a la rama superior. Véase que la rama superior tiene cuatro funciones para los intervalos que se muestran:

$$
\widetilde{W_{0}}(x)=\left\{\begin{array}{c}
\widetilde{W_{01}}(x),-\mathrm{e}^{-1} \leq x<1 \\
\widetilde{W_{02}}(x), 1 \leq x<40 \\
\widetilde{W_{03}}(x), 40 \leq x \leq 2000 \\
\widetilde{W_{04}}(x), 2000 \leq x
\end{array}\right.
$$

El subíndice -1 hace referencia a la rama inferior, la cual se constituye de 3 aproximaciones:

$$
\widetilde{W_{-1}}(x)=\left\{\begin{array}{l}
\widetilde{W_{-11}}(x),-\mathrm{e}^{-1} \leq x<-0.34 \\
\widetilde{W_{-12}}(x),-0.34 \leq x<-0.1, \\
\widetilde{W_{-13}}(x),-0.1 \leq x<-0.0001
\end{array}\right.
$$

Debido a la cantidad de términos algebraicos, al número de aproximaciones y al espacio del que se dispone, en el apéndice se presenta cada una de las de las aproximaciones 
para las dos ramas de la función Lambert $W$. Si se desea aumentar la exactitud de (6) y (7), se puede utilizar el algoritmo iterativo de Fritz

$$
\begin{gathered}
z_{n}=\ln \left(\frac{x}{W_{n}}\right)-W_{n}, \\
e_{n}=\left(\frac{z_{n}}{1+W_{m}}\right)\left(\frac{2\left(1+W_{n}\right)\left(1+W_{n}+\left(\frac{2}{3}\right)-z_{n}\right.}{2\left(1+W_{n}\right)\left(1+W_{n}+\left(\frac{2}{3}\right)-z_{n}\right.}\right), \\
W_{n+1}=W_{n}\left(1+e_{n}\right),
\end{gathered}
$$

donde $n$ es el número de iteraciones $e_{n}$. En Corless et al. (1996) y Vázquez-Leal et al. (2019) se presentan detalles del uso de este algoritmo.

\section{Funciones integrales de Fresnel}

Las aproximaciones que se proponen para las dos integrales de Fresnel que permiten evaluarlas están expresadas por

$$
\begin{aligned}
& \tilde{S}(x)=\left(-\frac{\cos \left(\frac{\pi|x|^{2}}{2}\right)}{\pi\left(|x|+16.73127745 \pi \mathrm{e}^{-1.5763886076 \pi \sqrt{|x|}}\right)}+\right. \\
& \frac{2}{25}\left(1-\mathrm{e}^{-1.714028381654 \pi|x|^{2}}\right)+\frac{1}{10}\left(1-\mathrm{e}^{-0.9 \pi|x|}\right) \operatorname{sgn}(x), \\
& \tilde{C}(x)=\left(-\frac{\sin \left(\frac{\pi|x|^{2}}{2}\right)}{\pi\left(|x|+20 \pi \mathrm{e}^{-200 \pi \sqrt{|x|}}\right)}+\frac{8}{25}\left(1-\mathrm{e}^{-0.69 \pi|x|^{3}}\right)\right. \\
& +\frac{2}{25}\left(1-\mathrm{e}^{-4.5 \pi|x|^{2}}\right)+\frac{1}{10}\left(1-\mathrm{e}^{-1.552940682 \pi|x|}\right) \operatorname{sgn}(x),
\end{aligned}
$$

(10)

para $-\infty<x<\infty$. 


\section{Revista Iberoamericana para la Investigación y el Desarrollo Educativo ISSN $2007-7467$}

En la figura 3 se presentan las integrales seno y coseno de Fresnel.

Figura 3. Integrales de Fresnel

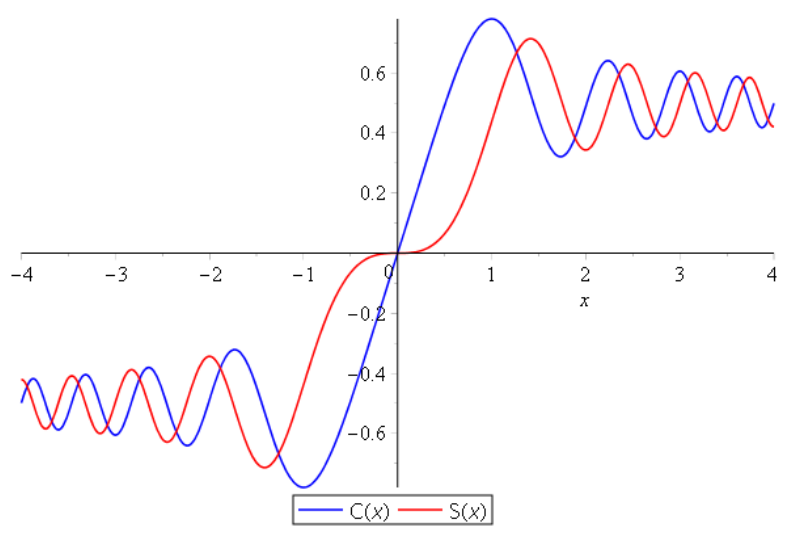

Fuente: Elaboración propia

\section{Función error}

Para la función error usaremos la expresión

$$
\begin{gathered}
\widetilde{\operatorname{erf}}(x)=\frac{2}{1+e^{\xi(x)}}-1-\infty<x<\infty \\
\xi(x)=\frac{-1}{5670}\left(\frac{105 \pi^{4}-9328 \pi^{3}+116928 \pi^{2}-483840 \pi+645120}{\sqrt{\pi^{9}}}\right) x^{9} \\
+\frac{2}{315}\left(\frac{15 \pi^{3}-532 \pi^{2}+3360 \pi-5760}{\sqrt{\pi^{7}}}\right) x^{7} \\
-\frac{2}{15}\left(\frac{3 \pi^{2}-40 \pi+96}{\sqrt{\pi^{5}}}\right) x^{5}+\frac{4}{3}\left(\frac{\pi-4}{\sqrt{\pi^{3}}}\right) x^{3}-\frac{4}{\sqrt{\pi}} x .
\end{gathered}
$$

A la función complementaria de esta función se le define como función error complementario erfc $(x)$, dado por $\operatorname{erfc}(x)=1-\operatorname{erf}(x)$.

En la figura 4 podemos ver que erf $(x)$ es una función impar. 
Figura 4. Función error

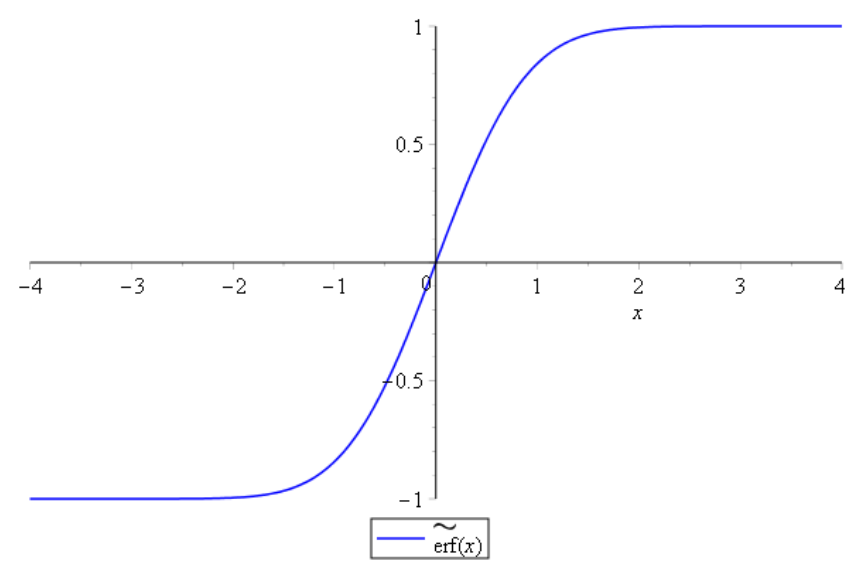

Fuente: Elaboración propia

\section{Función de distribución de probabilidad normal}

Para obtener el valor numérico de la probabilidad sin utilizar las clásicas tablas usaremos la aproximación

$$
\begin{gathered}
\tilde{P}(x)=\frac{1}{1+\mathrm{e}^{\zeta(x)}},-\infty<x<\infty \\
\zeta(x)=\frac{-\sqrt{2}}{181440}\left(\frac{105 \pi^{4}-9328 \pi^{3}+116928 \pi^{2}-483840 \pi+645120}{\sqrt{\pi^{9}}}\right) x^{9} \\
+\frac{\sqrt{2}}{2520}\left(\frac{15 \pi^{3}-532 \pi^{2}+3360 \pi-5760}{\sqrt{\pi^{7}}}\right) x^{7} \\
\frac{-\sqrt{2}}{60}\left(\frac{3 \pi^{2}-40 \pi+96}{\sqrt{\pi^{5}}}\right) x^{5}+\frac{\sqrt{2}}{3}\left(\frac{\pi-4}{\sqrt{\pi^{3}}}\right) x^{3}-\frac{2 \sqrt{2}}{\sqrt{\pi}} x .
\end{gathered}
$$

En la figura 5 se muestra su gráfica. 
Figura 5. Función de distribución acumulativa de probabilidad

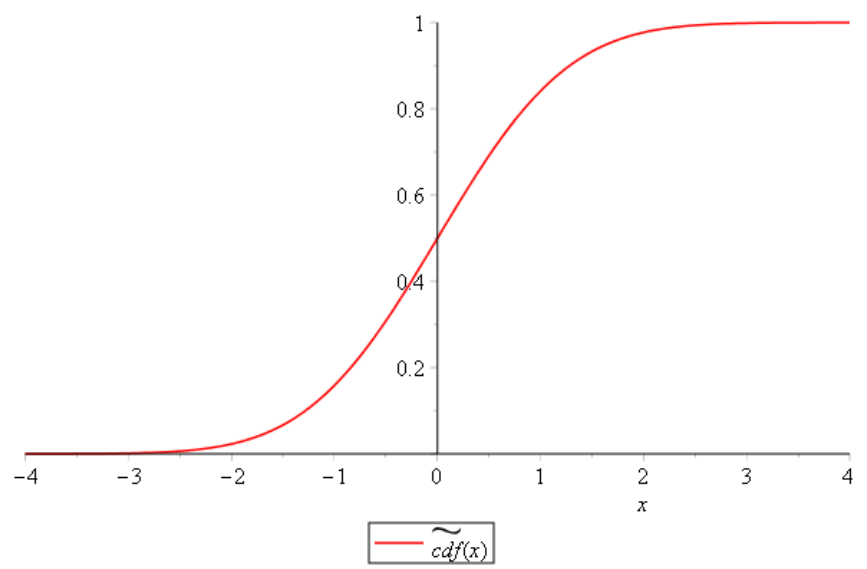

Fuente: Elaboración propia

\section{Funciones integrales completas elípticas de primera y segunda clase}

Para evaluar las integrales elípticas completas de primera y segunda clase presentamos:

$$
\begin{gathered}
\tilde{F}(x)=\frac{11}{7}+\ln \left(\frac{\sqrt[23]{\left(1-\frac{31}{47} x^{2}\right)^{2}} \sqrt[46]{\left(1-\frac{7}{38} x^{2}\right)^{9}}}{\sqrt[35]{\left(1-x^{2}\right)^{17}}}\right) \\
\tilde{E}(x)=\frac{360}{779} \sqrt{1-\frac{280}{339} x^{2}}+\frac{61}{118} \sqrt{1-\frac{245}{694} x^{2}}+ \\
\frac{159}{751} \sqrt{1-\frac{157}{157} x^{2}+\frac{19}{50} \sqrt{1-\frac{123}{4318} x^{2}}}
\end{gathered}
$$

En la figura 6 se ofrecen las gráficas de las funciones elípticas de primera y segunda clase. 
Figura 6. Funciones elípticas de primera y segunda clase

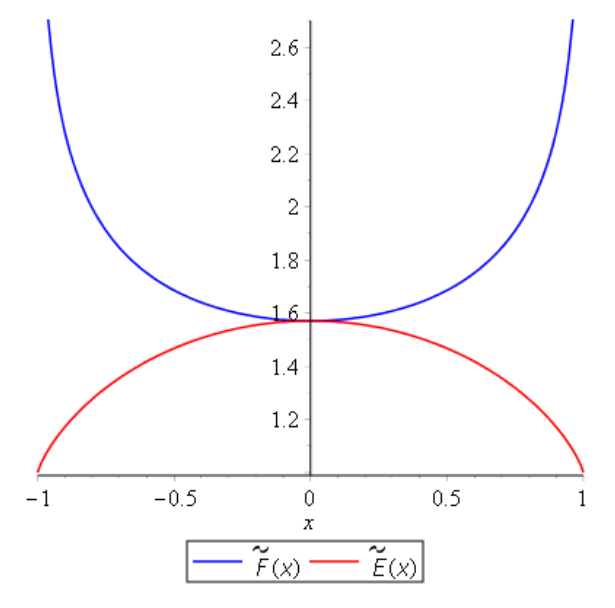

Fuente: Elaboración propia

\section{Función Gamma}

Para evaluar $\Gamma(x)$ debe utilizarse las aproximaciones dadas por

$$
\tilde{\Gamma}(x)=\left\{\begin{array}{c}
\widetilde{\Gamma_{1}}(x), x<0 \\
\widetilde{\Gamma_{2}}(x), 0<x \leq 2.043(15) \\
\widetilde{\Gamma_{3}}(x), x>2.043
\end{array}\right.
$$

con

$$
\begin{gathered}
\widetilde{\Gamma_{1}}(x)=\frac{\sqrt{2 \pi}\left(\frac{-\mathrm{e}}{x}\right)^{-x}}{2 \sqrt{-x} \sin (\pi x)}\left(1-0.340198059 \mathrm{e}^{7.511328218 x}-\right. \\
\left(0.259363253 \mathrm{e}^{1.320358968 x}\right), x<0 \\
\widetilde{\Gamma_{2}}(x)=\frac{1}{\mathrm{e}^{\varphi(x)}-1}, 0<x \leq 2.043 \\
\varphi(x)=0.2084721215 x \mathrm{e}^{-0.6941829682 x^{2}+} \\
0.6902220099 x \mathrm{e}^{-0.1818111523 x^{2}}+0.08383862837 \mathrm{e}^{-1.832856342 x^{2}} \\
\widetilde{\Gamma_{3}}(x)=\sqrt{2 \pi(x-1)}\left(\frac{x-1}{\mathrm{e}}\right)^{x-1}\left(1+\frac{1}{r(x)}\right)^{\left(\frac{1}{12}\right)} \mathrm{e}^{\left(-\frac{7}{\varepsilon(x)}-\frac{1531}{\zeta(x)}\right)}, x>2.043,
\end{gathered}
$$




$$
\begin{gathered}
\varepsilon(x)=720(x-1)^{3}-\frac{900}{49}(x-1) \\
\zeta(x)=1975680(x-1)^{7}+\frac{34595736000}{16841}(x-1)^{5}- \\
\frac{10219256619062120}{3687050653}(x-1)^{3}+ \\
\frac{1556259293563438478000}{186280860141519}(x-1) \\
r(x)=x-\frac{3}{2}+(7.664112723(x-1)) \mathrm{e}^{-7.787686838(x-1)}+ \\
+(0.0223051314(x-1)) \mathrm{e}^{-4.708891117(x-1)}
\end{gathered}
$$

En la figura 7 se presenta la gráfica $\Gamma(x)$ para $-\infty<x<\infty$ en el dominio de los números reales.

Figura 7. Función $\Gamma(x)$ aproximada

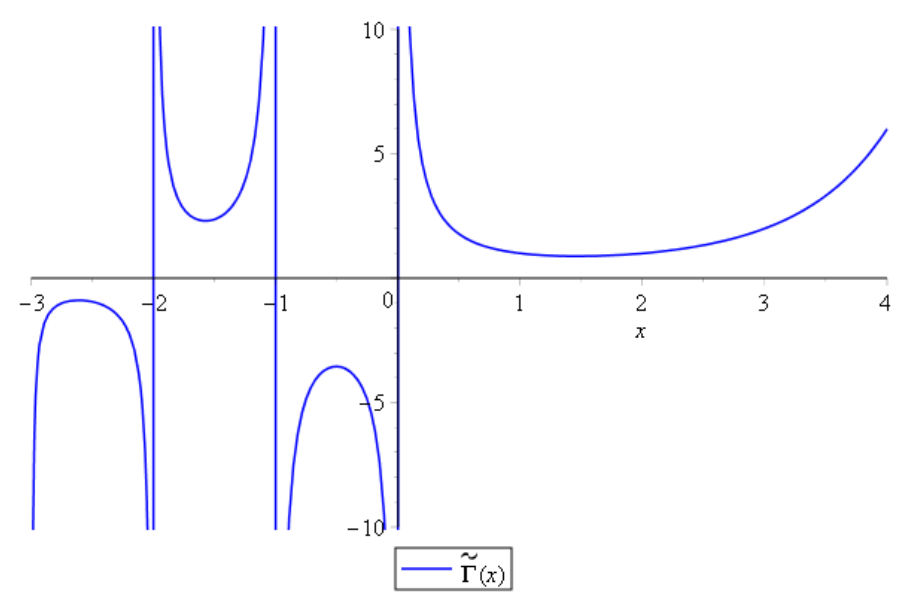

Fuente: Elaboración propia

\section{Casos de estudio}

Ejemplo 1. Determinar la corriente de lazo en un circuito con dos diodos en serie

En los cursos de circuitos electrónicos analógicos se estudia la polarización del diodo rectificador utilizando un circuito con un diodo, una resistencia y una fuente de voltaje (Boylestad y Nashelsky, 2009). De manera tradicional se determina el punto de polarización 
del diodo donde se cortan la recta de carga del circuito y la curva del diodo rectificador; sin embargo, es posible determinar este punto solución utilizando algún algoritmo numérico como Newton Raphson (Burden y Faires, 1996).

En Sandoval-Hernández et al. (2019b) se propuso hallar la solución de este circuito utilizando dos diodos rectificadores en serie con una sola fuente de voltaje independiente y una resistencia con valores numéricos para cada elemento de $V=3 V, R=5 \Omega, I_{s 1}=1 E-$ $12, I_{S 2}=1 E-9, V_{T}=25.86 \mathrm{~m} V$, mediante el método de perturbación clásico (figura 8 ). La solución para el punto de polarización obtenida en Sandoval-Hernández et al. (2019b) fue algebraica, en términos de los parámetros del circuito. En este artículo procederemos a mostrar una alternativa diferente de solución para este circuito con el fin de superar el DME utilizando para ello la función Lambert W.

Figura 8. Circuitos con dos diodos rectificadores en serie

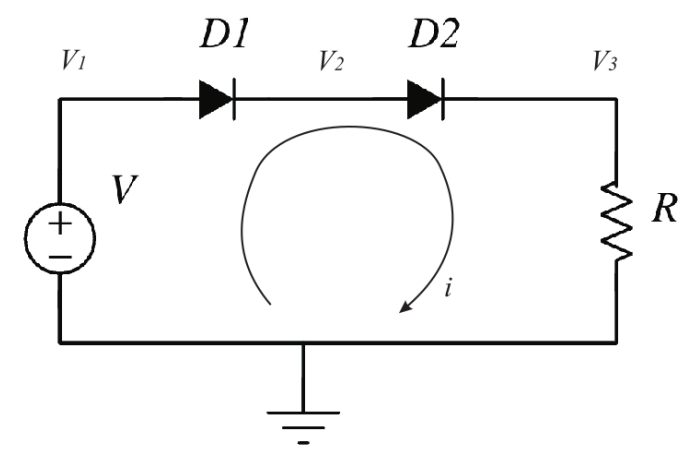

Fuente: Sandoval-Hernández et al. (2019b)

Solución: Se asume que los diodos tienen diferentes corrientes de saturación. Utilizando la ley de las tensiones de Kirchoff (LVK) Alexander y Sadiku (2013), se tiene lo siguiente:

$$
R i+2 V_{T} \ln (i)-V_{T} \ln \left(I_{s 1}\right)-V_{T} \ln \left(I_{s 2}\right)-V=0,
$$

donde $V_{T}$ es voltaje térmico, $I_{S 1}, I_{S 2}$ son las corrientes de saturación en cada uno de los diodos.

La ecuación (19) es no lineal y no se puede resolver utilizando los métodos algebraicos convencionales. Utilizaremos la función Lambert W para resolver.

$$
R i+2 V_{T} \ln (i) V_{T} \ln \left(I_{s 1} I_{s 2}\right)+V \cdot(20)
$$



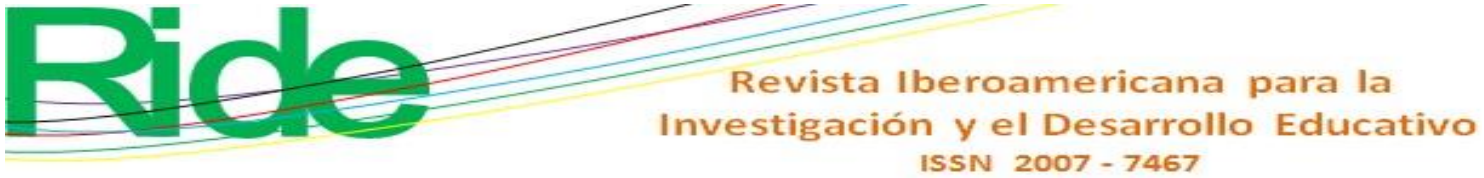

Para simplificar los cálculos algebraicos hacemos $K=V_{T} \ln \left(I_{s 1} I_{s 2}\right)+V$ en (20) para obtener

$$
R i+2 V_{T} \ln (i)=K
$$

Resolviendo algebraicamente, tenemos

$$
\frac{-2 V_{T} \ln (i)+K}{R i}=1
$$

Multiplicando (22) por $\mathrm{e}^{\frac{K}{2 V_{T}}}$,

$$
\left(\frac{-2 V_{T} \ln (i)+K}{R i}\right) \mathrm{e}^{\frac{K}{2 V_{T}}}=\mathrm{e}^{\frac{K}{2 V_{T}}} .
$$

Manipulando los términos algebraicamente

$$
\left(-\ln (i)+\frac{K}{2 V_{T}}\right) \mathrm{e}^{-\ln (i)+\frac{K}{2 V_{T}}}=\frac{R}{2 V_{T}} \mathrm{e}^{\frac{K}{2 V_{T}}}
$$

Aplicando la función de Lambert W y despejando $i$ y sustituyendo el valor de $K$, tenemos la expresión algebraica en función de todos los parámetros del circuito para encontrar analíticamente la corriente del circuito dado por

$$
i=\mathrm{e}^{\frac{-1}{2 V_{T}}\left[2 V_{T} W\left(\frac{R}{2 V_{T}} \mathrm{e}^{\frac{V_{T} \ln \left(I_{S 1} I_{S 2}\right)+V}{2 V_{T}}}\right)-V_{T} \ln \left(I_{S 1} I_{S 2}\right)-V\right]} .
$$

Utilizando (6) y sustituyendo los valores dados para cada elemento del circuito en (25) obtenemos el valor de $i=0.3604662014$ A.

Ejemplo 2. Factorial de un número con punto decimal o negativo

En los libros de texto para estadística (Alanís-Martínez, 2016; Espinoza-Casares, 2017) del bachillerato tecnológico tenemos que el factorial de número entero positivo $n$ se representa por $n$ !. Sin embargo, no se dice nada acerca del factorial para números con punto decimal o negativos. La función $\Gamma(x)$ nos ayuda a resolver este problema. Nosotros hallaremos el factorial de -3.79 utilizando (15). Esto es, $(-3.79) !=0.2975244944$. 
Ejemplo 3. Inductancia mutua de dos bobinas paralelas

En Nalty (2011) se calculó la inductancia mutua de dos bobinas separadas a lo largo del eje $z$, a una distancia $\frac{l}{2}=0.5 m$ del origen, con radio $L=R=0.5 m$, con permitidad magnética $\mu=4 \pi E-7 N A^{-2}$, con inductancia mutua de $M=7.093 E-8 H$. En la figura 9 se presenta el diagrama de inductancia mutua de dos bobinas coaxiales.

Figura 9. Inductancia mutua de 2 bobinas paralelas
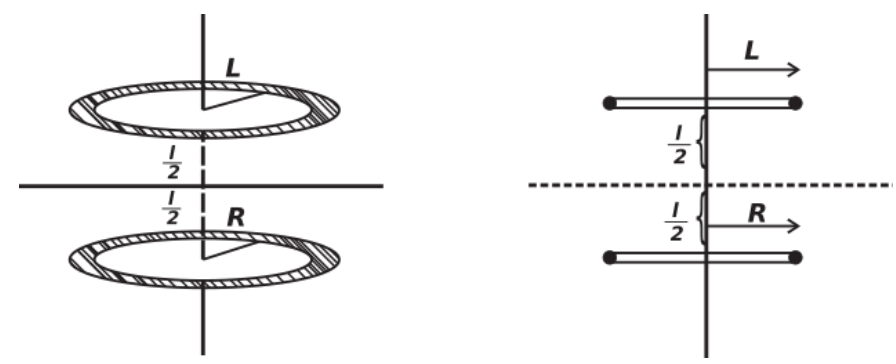

Fuente: Elaboración propia

La ecuación que permite hallar la inductancia mutua de esta configuración está dada por

$$
M=2 \mu \frac{a+b}{b}\left(\left(1-\frac{\beta^{2}}{2}\right) F(x)-E(x)\right)
$$

con

$$
a=\frac{L^{2}+R^{2}+l^{2}}{(L R)^{2}}, b=\frac{2}{L R}, x=\sqrt{\frac{2 b}{a+b}} .
$$

Sustituyendo los valores numéricos en (26) y (27) y utilizando $\tilde{F}(x)$ y $\tilde{E}(x)$, se tiene que la inductancia mutua $M=7.092 E-8 H$.

Ejemplo 4. Determinación de la probabilidad en una población

En Wayne (2000) se calculó la probabilidad de este caso de estudio utilizando las tablas clásicas para la distribución normal. Nosotros utilizaremos (16) para determinarla.

El peso de una muestra poblacional tiene aproximadamente una distribución normal con una de media de 140 libras y desviación estándar de 25 libras. ¿Cuál es la probabilidad de seleccionar al azar a una persona con un peso dentro del rango de 100 y 170 libras?

Solución: Sea la transformación para tipificar 


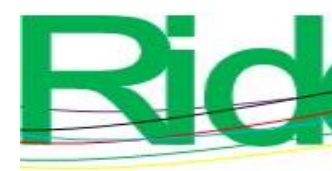

$$
\begin{aligned}
& \text { Revista Iberoamericana para la } \\
& \text { Investigación y el Desarrollo Educativo } \\
& \text { ISSN } 2007-7467 \\
& Z=\frac{X-\mu}{\sigma} \text {, (28) }
\end{aligned}
$$

donde $X$ es la variable aleatoria del peso de la población, $\mu$ es la media, $\sigma$ la desviación estándar. Sustituyendo $X=100,170 \mathrm{lb}, \mu=140 \mathrm{lb}, \sigma=25 \mathrm{lb}$ en 38 , se tiene $z_{1}=-1.6, z_{2}=$ 1.2 . Sustituyendo en $(12)$, se tiene que $\widetilde{P_{1}}(-1.6)=0.0548628902, \widetilde{P_{2}}(1.2)=$ 0.884768632 , respectivamente. Consecuentemente, la probabilidad $\tilde{P}(-1.6 \leq z \leq 1.2)=$ 0.830013973 .

\section{Limitaciones}

Para implementar esta propuesta curricular es necesario que los profesores que imparten las asignaturas de matemáticas cuenten con el perfil académico afín al campo disciplinar de matemáticas, de acuerdo a la selección del profesorado por la Unidad del Sistema para la Carrera de las Maestras y Maestros, es decir, deben poseer una formación académica universitaria y docente necesaria que les permita diseñar y aplicar otras estrategias didácticas de manera adicional a las que existen actualmente en la enseñanza de las funciones. De esta manera, los facilitadores podrán recuperar de sus respectivos campos profesionales (química, física, así como ingeniería mecánica, eléctrica, civil, etc.) la aplicación de estas funciones y proponerlas en el aula a los alumnos con el fin de propiciar el aprendizaje significativo (Arceo et al., 2010; Charur, 2016; Morales-Lizama, 2016).

\section{Conclusiones}

El objetivo de este trabajo fue presentar una propuesta curricular para la asignatura propedéutica de matemáticas aplicadas del bachillerato tecnológico con la finalidad de reforzar la enseñanza de las funciones y sus características. En tal sentido, a conceptos como dominio, rango, valor absoluto, periodicidad y paridad, entre otros, se les ha dado mucha importancia, ya que su comprensión es necesaria para estudiar el comportamiento de las funciones matemáticas.

Ahora bien, se propone la adición de la función Lambert $\mathrm{W}$ a las funciones trascendentes, ya que pertenece a este grupo de funciones y, hasta la fecha, no se le ha dado su lugar en los libros de texto. Esta función posee propiedades algebraicas que ayudan a realizar otros despejes algebraicos que no se presentan en los libros tradiciones de álgebra y 


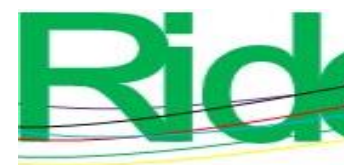

Revista Iberoamericana para la Investigación y el Desarrollo Educativo ISSN 2007 - 7467

precálculo. Asimismo, se propone por primera vez agregar en los planes de estudio la incorporación de las funciones hiperbólicas y especiales, las cuales tienen muchas aplicaciones en la ingeniería que se encuentra en el cotidiano del estudiante, como en los fundamentos de los aparatos electrodomésticos, los fenómenos físicos, las construcciones, etc.

Para el estudio de las funciones especiales se ha presentado una serie de aproximaciones que se hallan en términos de expresiones elementales haciendo posible estudiarlas en el salón de clase y evitando el posible uso de software matemático que incorpora rutinas interconstruidas que lo harían ver como una caja negra.

Sin embargo, para implementar esta propuesta curricular es necesario que los docentes del área de matemáticas posean una buena formación académica y pedagógica, y sean entusiastas para indagar en ejemplos prácticos aplicativos en el cotidiano del alumno. De esta manera, cada facilitador podrá aprovechar las estrategias didácticas necesarias utilizando sus competencias docentes para hacer significativo los conocimientos que adquirirán los alumnos.

Consideramos que el estudio de las funciones especiales e hiperbólicas enriquecerá la perspectiva del estudiante del bachillerato tecnológico referente al cotidiano que lo rodea porque el uso de las funciones no se limita exclusivamente a los problemas aritméticos y algebraicos que se le han presentado de manera tradicional dentro del aula. Por eso, se espera que la presente propuesta incentive a los estudiantes a estudiar una licenciatura relacionada con las ciencias exactas y de ingeniería, y que en su momento contribuyan al desarrollo y progreso tecnológico de nuestro país.

\section{Futuras Líneas de Investigación}

Se deben diseñar estrategias didácticas piloto que permitan implementar la propuesta didáctica presentada en este trabajo con el fin de llevarla a cabo, sin perder de vista los conocimientos previos que posean los alumnos sobre el manejo y graficado de las funciones elementales. Además, es importante efectuar un análisis estadístico de los aprendizajes esperados en la prueba piloto para realizar las posibles adecuaciones. Para ello, también será necesario diseñar los instrumentos de evaluación para recolectar la información que deseamos obtener. Asimismo, es importante sugerir a los diferentes autores que publican 

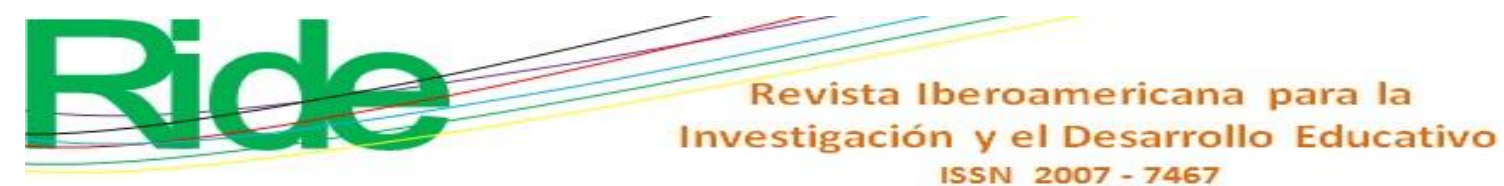

libros de texto del nivel medio superior que incluyan las funciones propuestas en este trabajo con el fin de promover su enseñanza en este nivel educativo.

Esta propuesta curricular, en definitiva, se puede extender a los primeros semestres de la educación universitaria, donde se retoman conceptos de precálculo vistos en el bachillerato. Muchos alumnos que ingresan a las instituciones universitarias y que aprueban el primer semestre continúan desconociendo la existencia de las funciones especiales.

\section{Referencias}

Abramowitz, M., Stegun, I. A. y Romer, R. H. (1988). Handbook of mathematical functions with formulas, graphs, and mathematical table.

Alanís-Martínez, M. A., (2016). Probabilidad y estadística. Fondo de Cultura Económica, Colección Dgeti.

Alexander, C. K., y Sadiku, M. N. (2013). Fundamentos de circuitos eléctricos. AMGH Editora.

Almenar, M. E., Isla, F., Gutiérrez, S. y Luege, M. (2018). Programa de elementos finitos de código abierto para la resolución de problemas mecánicos en estado plano. Asociación Argentina de Mecánica Computacional, 36, 6-9.

Álvarez, R. B. y Mayo, I. C. (2009). Las tecnologías de la información y la comunicación en la educación superior. Estudio descriptivo y de revisión. Revista Iberoamericana de Educación, 50(7), 1-12.

Arceo, F. D. B., Rojas, G. H. y González, E. L. G. (2010). Estrategias docentes para un aprendizaje significativo: una interpretación constructivista. McGraw-Hill Interamericana.

Ayres, F., Mendelson, E. y Abellanas, L. (1994). Cálculo diferencial e integral. Serie Schaum McGraw-Hill.

Balassone, V. and Romero, H. (2015). Determination of the solution of the integral double of Fresnel to predict the electromagnetic attenuation in the case of double knife edges with ground reflexion. Revista Técnica de la Facultad de Ingeniera Universidad del Zulia, 38(1), 83-91.

Baldor, J. A. (2011). Álgebra. Editorial Patria.

Barnett, R. (1994). Álgebra con geometría analítica y trigonometría. México: LimusaNoriega. 


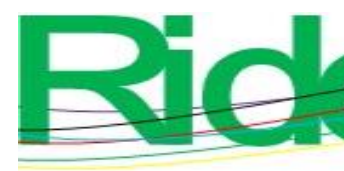

\section{Revista Iberoamericana para la Investigación y el Desarrollo Educativo ISSN 2007 - 7467}

Bermúdez, C. (2005). Curso básico de estructuras metálicas. Manizales, Colombia: Universidad Nacional de Manizales.

Bird, R. B., Stewart, W. y Lightfoot, E. (2002). Transport Phenomena. New York: John Wiley \& Sons.

Boylestad, R. y Nashelsky, L. (2009). Teoría de circuitos. Prentice Hall-Pearson Educación.

Brizard, A. J. (2009). A primer on elliptic functions with applications in classical mechanics. European Journal of Physics, 30(4), 729.

Burden, R. L. y Faires, J. D. (1996). Análisis numérico (2. ${ }^{\mathrm{a}}$ ed.). Grupo Editorial Iberoamérica.

Casares, J. L. (2018). Cálculo diferencial. México: Fondo de Cultura Económica. Colección DGETI.

Charur, C. Z. (2016). Instrumentación didáctica por competencias. Grupo Editorial Patria.

Collado, E. C., Huallapacusi, M. y Osores, O. (2005). Sistema de control de temperatura para una reacción de saponificación en un reactor químico continuo enchaquetado. Tecnia, 15(1).

Cordero Osorio, F. (2002). Reconstrucción de significados en contextos interactivos: las gráficas de las funciones en la organización del cálculo. Acta Latinoamericana de Matemática Educativa, 15(2), 815-820.

Cordero Osorio, F. y Domínguez García, I. (2001). Algunas construcciones de comportamientos asintóticos senoidales en estudiantes de precálculo y cálculo. Acta Latinoamericana de Matemática Educativa, 14, 318-325.

Cordero, F. (2013). Matemáticas y el cotidiano. Diplomado desarrollo de estrategias de aprendizaje para las matemáticas del bachillerato: la transversalidad curricular de las matemáticas. Módulo III. Cinvestav-IPN.

Cordero, F. y Suárez, L. (2005). Modelación en matemática educativa. Acta Latinoamericana de Matemática Educativa, 18,639-644.

Corless, R. M., Gonnet, G. H., Hare, D. E., Jeffrey, D. J. y Knuth, D. E. (1996). On the Lambert W function. Advances in Computational mathematics, 5(1), 329-359.

Diario Oficial de la Federación [DOF] (2004). Acuerdo número 345 por el que se establece el Plan de Estudios el bachillerato tecnológico. Diario Oficial de la Federación.

Diario Oficial de la Federación [DOF] (2008). Acuerdo número 444 por el que se establecen las competencias que constituyen el Marco Curricular Común del Sistema Nacional de 


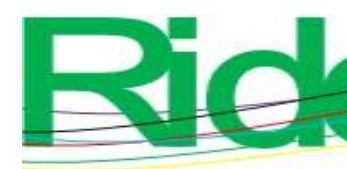

Revista Iberoamericana para la Investigación y el Desarrollo Educativo ISSN 2007 - 7467

Bachillerato el Plan de Estudios el bachillerato tecnológico. Diario Oficial de la Federación.

Diario Oficial de la Federación [DOF] (2012). Acuerdo número 653 por el que se establece el Plan de Estudios el bachillerato tecnológico. Diario Oficial de la Federación.

Edwards, C. H. y Penney, D. E. (2011). Ecuaciones diferenciales y problemas con valores en la frontera. Pearson Educación.

Espinoza-Casares, J. L. (2017). Probabilidad y estadística (2. ${ }^{\mathrm{a}}$ ed.). Fondo de Cultura Económica, Colección Dgeti.

Filobello-Nino, U., Vázquez-Leal, H., Pérez-Sesma, J. A. A., Pérez-Sesma, A., SandovalHernández, M. et al. (2017b). Classical perturbation method for the solution of a model of diffusion and reaction. American Scientific Research Journal for Engineering, Technology, and Sciences (ASRJETS), 27(1), 151-160.

Filobello-Nino, U., Vazquez-Leal, H., Sandoval-Hernandez, M. et al. (2017a). A high accurate approximation for a galactic newtonian nonlinear model validated by employing observational data. American Scientific Research Journal for Engineering, Technology, and Sciences (ASRJETS), 27(1), 139-150.

Fox, W. P. (2011). Mathematical modeling with Maple. Nelson Education.

Fukushima, T. y Kopeikin, S. (2014). Elliptic functions and elliptic integrals for celestial mechanics and dynamical astronomy. Frontiers in Relativistic Celestial Mechanics, 2, $189-228$.

González, R. C. y Woods, R. E. (1996). Tratamiento digital de imágenes (3. a ed.). New York: Addison-Wesley.

Greenhill, A. G. (1907). The elliptic integral in electromagnetic theory. Transactions of the American Mathematical Society, 8(4), 447-534.

Haykin, S. (1994). An introduction to analog and digital communication. John Wiley \& Sons. Hecht, E. and Zajac, A. (1987). Optics. Massachusetts.

Hernández-Sampieri, R., Fernández-Collado, C. y Baptista, L. P. (2010). Metodología de la investigación. McGraw-Hill Interamericana.

Johansson, F. (2020). Computing the Lambert W function in arbitrary-precision complex interval arithmetic. Numerical Algorithms, 83(1), 221-242.

Komla-Amenyou, F. (2018). Properties and Computation of the inverse of the Gamma Function, The University of Western Ontario. 


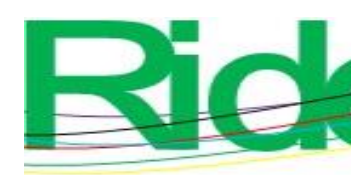

Revista Iberoamericana para la

Investigación y el Desarrollo Educativo

ISSN 2007 - 7467

Kindle, J. (1994). Geometría analítica. McGraw Hill-Serie Shaum.

Leithold, L. (2012). EC7 Cálculo. Oxford.

Lie, K. A. (2019). An introduction to reservoir simulation using MATLAB/GNU OCTAVE. Cambridge University Press.

Mora-Sánchez, J. A. (2019). Investigaciones en clase de matemáticas con GeoGebra. XIII jornades d'Educació Matemàtica de la Comunittat Valenciana: Innovació i tecnologia en educació matemàtica. Instituto de Ciencias de la Educación.

Morales-Lizama, F., (2016). Desarrollo de competencias educativas: guía para la elaboración de secuencias didácticas y orientación a la planeación didáctica argumentada. Trillas.

Nalty, K. (2011). Classical calculation for mutual inductance of two coaxial loops in MKS units. Austin, TX, 78750, 1-8.

Ogata, K. (2003). Ingeniería de control moderna. Pearson Educación.

Oldham, K. B., Myland, J. and Spanier, J. (2010). An atlas of functions: with equator, the atlas function calculator. Springer Science \& Business Media.

Olguín-Díaz, C. y Sánchez-Linares, I. (2016). Guía de preparación para la prueba planea MS. Gafra.

Olvera, B. G. (1991). Geometría analítica. Colección DGETI.

Proakis, J. G. y Masoud, S. (2001). Digital communications. McGraw-Hill.

Purcell, E J., Rigdon, S. E. y Varbeg, D. E. (2007). Cálculo (9. a ed.). Pearson Educación Prentice Hall.

Sadiku, A. M., (1998). Electromagnetismo. México: CECSA.

Sandoval-Hernández, M. A., Álvarez-Gasca, O., Contreras-Hernández, A. D. et al. (2019b). Exploring the classic perturbation method for obtaining single and multiple solutions of nonlinear algebraic problems with application to microelectronic circuits. International Journal of Engineering Research \& Technology, 8(9).

Sandoval-Hernández, M. A., Vázquez-Leal, H., Filobello-Nino, U. y Hernández-Martínez, L. (2019a). New handy and accurate approximation for the Gaussian integrals with applications to science and engineering. Open Mathematics, 17(1), 1774-1793.

Sandoval-Hernández, M., Vázquez-Leal, H., Hernández-Martínez, Filobello-Nino U. A., Jiménez-Fernández, V.M. Herrera-May A.L., Castaneda-Sheissa, Ambrosio-Lazaro, 


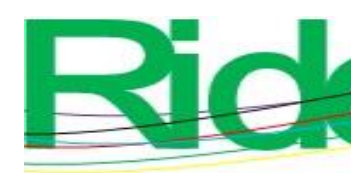

Revista Iberoamericana para la
Investigación y el Desarrollo Educativo
ISSN $2007-7467$

R.C. y Díaz-Arango G. U. (2018). Approximation of Fresnel integrals with applications to diffraction problems. Mathematical Problems in Engineering, Hindawi.

Serway R. A. y Jewett, J. W. (2018). Physics for scientists and engineers with modern physics. Cenange Learning.

Soto, D. y Cantoral, R. (2014). Discurso matemático escolar y exclusión. Una visión socioepistemológica. Bolema: Boletim de Educação Matemática, 28(50), 1525-1544.

Soto, D., Gómez, K., Silva, H. y Cordero, F. (2012). Exclusión, cotidiano e identidad: una problemática fundamental del aprendizaje de la matemática. Comité Latinoamericano de Matemática Educativa, 1041-1048.

Stewart, J. (2015). Single variable calculus: Early trascendentals. Cengage Learning.

Torres-Remon, M., (2016). Aplicaciones con VBA con Excel. Alfaomega.

Treviño, E., Alcocer, S., Flores, L., Larrúa, R., Zárate, J. y Gallegos, L. (2004). Investigación experimental del comportamiento de muros de mampostería confinada de bloques de concreto sometidos a cargas laterales cíclicas reversibles reforzados con acero de grados 60 y 42. En XIV Congreso Nacional de Ingeniería Estructural. Acapulco: Sociedad Mexicana de Ingeniería Estructural ISME.

Uriza, R. C. (2000). Desarrollo del pensamiento matemático. Trillas.

Uriza, R. C., Espinosa, G. M. y Gasperini, D. R. (2015). Análisis del discurso matemático escolar en los libros de texto: una mirada desde la teoría socioepistemológica. Avances de Investigación en Educación Matemática, 8, 9-28.

Vázquez-Leal, H. y Sarmiento-Reyes, A. (2015). Power series extender method for the solution of nonlinear differential equations. Mathematical Problems in Engineering, 2015.

Vázquez-Leal, H., Filobello-Nino, U., Sarmiento-Reyes, A., Sandoval-Hernández, M., Pérez-Sesma, J. A. A., Pérez-Sesma, A., Jiménez-Fernández, V. M., Huerta-Chúa, J., Castro-González, F., Sánchez-Orea, J., Hernández-Machuca, S.F., Cuellar-Hernández, L., Pretelin-Canela, J. E., Gasca-Herrera, A. E., Sampieri-González, C. E., PalmaGrayeb, B. E., Contreras-Hernández, A. D., Álvarez-Gasca O., y González-Martínez, F. J. (2017). Application of HPM to Solve Unsteady Squeezing Flow of a Second-Grade Fluid between Circular Plates. American Scientific Research Journal for Engineering, Technology, and Sciences (ASRJETS), 27(1), 161-178. 
Vázquez-Leal, H., Sandoval-Hernández, M. A., y Filobello-Nino, U. (2020). The novel family of transcendental Leal-functions with applications to science and engineering. Heliyon, 6(11).

Vázquez-Leal, H., Sandoval-Hernández, M. A., García-Gervacio, J. L., Herrera-May, A. L. y Filobello-Nino, U. A. (2019). PSEM approximations for both branches of lambert function with applications. Discrete Dynamics in Nature and Society, 2019.

Vázquez-Leal, H., Sandoval-Hernández, M., Castañeda-Sheissa, Filobello-Nino, U. y Sarmiento-Reyes, A. (2015b). Modified Taylor solution of equation of oxygen diffusion in a spherical cell with Michaelis-Menten uptake kinetics. International Journal of Applied Mathematics Research, 4(2), 253.

Wayne, D. (2000). Bioestadística. Base para el análisis de las ciencias de la salud (3. ${ }^{\mathrm{a}}$ ed.). UTHEA.

Wazwaz, A. M. (2011). Linear and nonlinear integral equations. Berlín, Springer.

Zhen-Han, Y., y Jing-Feng, T., (2018). An accurate approximation formula for gamma function, Journal of Inequalities and Applications, Springer, 1-9.

Zill, D. G. (1997). Ecuaciones diferenciales con aplicaciones. Editorial Iberoamérica. 


\begin{tabular}{|l|l|}
\hline Rol de Contribución & Autor (es) \\
\hline Conceptualización & Mario Alberto Sandoval Hernandez \\
\hline Metodología & Mario Alberto Sandoval Hernandez \\
\hline Software & Sergio Hernandez Mendez \\
\hline Validación & Salvador Enrique Torreblanca Bouchan \\
\hline Análisis Formal & Sergio Hernandez Mendez \\
\hline Investigación & Salvador Enrique Torreblanca Bouchan \\
\hline Recursos & Gerardo Ulises Diaz Arango \\
\hline Curación de datos & Gerardo Ulises Diaz Arango \\
\hline $\begin{array}{l}\text { Escritura - Preparación del } \\
\text { borrador original }\end{array}$ & Mario Alberto Sandoval Hernandez \\
\hline $\begin{array}{l}\text { Escritura - Revisión y } \\
\text { edición }\end{array}$ & Mario Alberto Sandoval Hernandez \\
\hline Visualización & Sergio Hernandez Mendez \\
\hline Supervisión & Mario Alberto Sandoval Hernandez \\
\hline Administración de Proyectos & Mario Alberto Sandoval Hernandez \\
\hline Adquisición de fondos & Mario Alberto Sandoval Hernandez \\
\hline
\end{tabular}




\section{Apéndice}

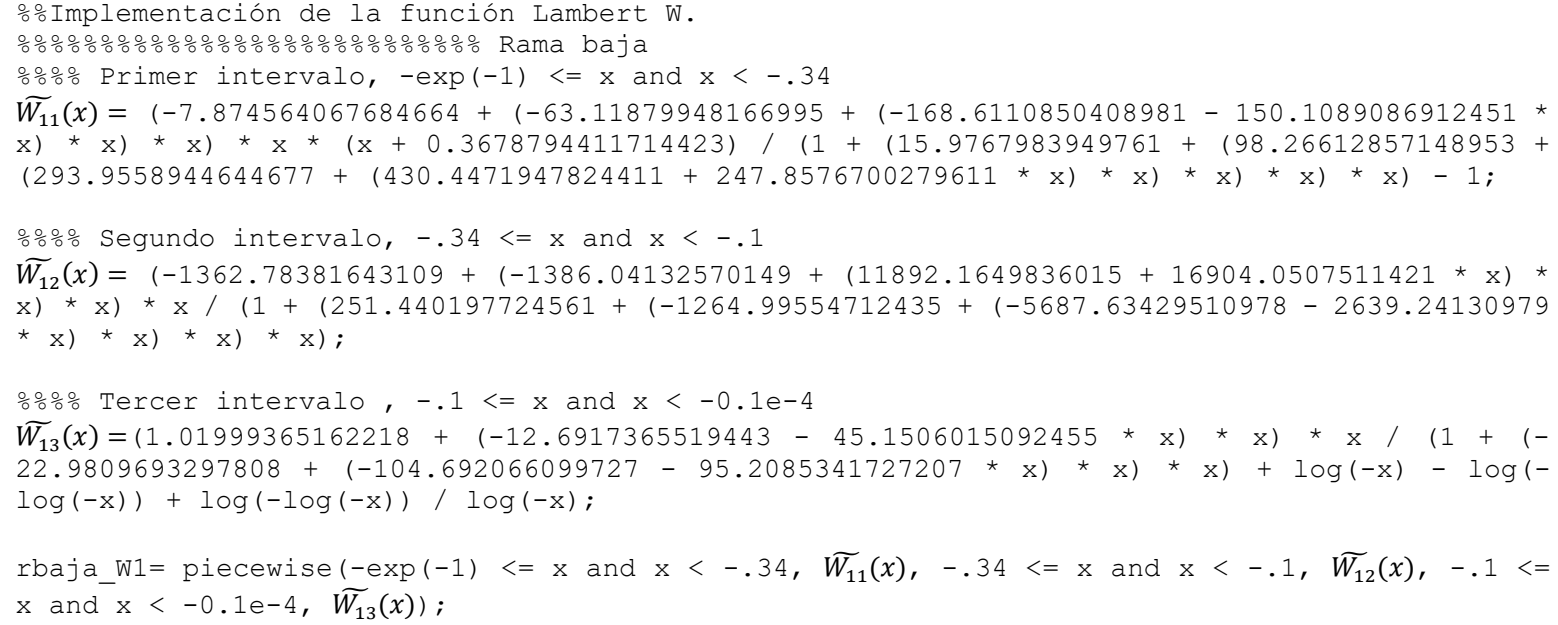

Nota: La instrucción piecewise se utiliza para concatenar las diferentes aproximaciones especificando el intervalo donde será válido. Esta instrucción se deberá modificar o quitar dependiendo del software donde se implemente. 